\title{
Determination of the Intrinsic Defect at the Origin of Poor H Evolution Performance of Monoclinic BivO Photocatalyst Using Density Functional Theory
}

Sheikha Lardhi, Luigi Cavallo, and Moussab Harb

J. Phys. Chem. C, Just Accepted Manuscript • DOI: 10.1021/acs.jpcc.8b03044 • Publication Date (Web): 27 Jul 2018

Downloaded from http://pubs.acs.org on July 31, 2018

\section{Just Accepted}

"Just Accepted" manuscripts have been peer-reviewed and accepted for publication. They are posted online prior to technical editing, formatting for publication and author proofing. The American Chemical Society provides "Just Accepted" as a service to the research community to expedite the dissemination of scientific material as soon as possible after acceptance. "Just Accepted" manuscripts appear in full in PDF format accompanied by an HTML abstract. "Just Accepted" manuscripts have been fully peer reviewed, but should not be considered the official version of record. They are citable by the Digital Object Identifier (DOI®). "Just Accepted" is an optional service offered to authors. Therefore, the "Just Accepted" Web site may not include all articles that will be published in the journal. After a manuscript is technically edited and formatted, it will be removed from the "Just Accepted" Web site and published as an ASAP article. Note that technical editing may introduce minor changes to the manuscript text and/or graphics which could affect content, and all legal disclaimers and ethical guidelines that apply to the journal pertain. ACS cannot be held responsible for errors or consequences arising from the use of information contained in these "Just Accepted" manuscripts. 


\title{
Determination of the Intrinsic Defect at the Origin of Poor $\mathrm{H}_{2}$
}

\section{Evolution Performance of Monoclinic $\mathrm{BiVO}_{4}$ Photocatalyst}

\section{Using Density Functional Theory}

\author{
Sheikha Lardhi, Luigi Cavallo, and Moussab Harb* \\ King Abdullah University of Science and Technology (KAUST), KAUST Catalysis Center \\ (KCC), Physical Sciences and Engineering Division (PSE), Thuwal, 23955-6900, Saudi Arabia
}




\begin{abstract}
The effects of intrinsic defects in monoclinic bismuth vanadate $\left(\mathrm{BiVO}_{4}\right)$ on its stability and optoelectronic properties for photochemical water splitting application were examined using density functional theory (DFT). Among the most favorable structures, only that associated with $\mathrm{V}$-antisites on $\mathrm{Bi}$ with additional Bi-vacancies $\left(\mathrm{Bi}_{(1-5 \mathrm{x})} \mathrm{V}_{(1+3 \mathrm{x})} \mathrm{O}_{4}\right.$ with $\left.x=0.0625\right)$ revealed narrower band gap energy by $0.5 \mathrm{eV}$ compared to pristine material (calculated value is $2.8 \mathrm{eV}$ ) giving a value of $2.3 \mathrm{eV}$, which is very close to the experimentally reported ones (in the 2.4-2.5 $\mathrm{eV}$ range). The low electron mobility reported experimentally for this material was also confirmed by the relatively large electron effective masses obtained for the intrinsic defective $\mathrm{Bi}_{(1-5 \mathrm{x})} \mathrm{V}_{(1+3 \mathrm{x})} \mathrm{O}_{4}(x=0.0625)$ structure along the three principal crystallographic directions. The strongly localized nature of the accommodated electrons on the d-orbitals of the newly substituted $\mathrm{V}$ at $\mathrm{Bi}$ sites was also predicted to be at the origin of the poor $\mathrm{H}_{2}$ evolution performance of this material.
\end{abstract}




\section{Introduction}

$\mathrm{BiVO}_{4}$ is visible-light driven semiconductor photocatalyst used in a large variety of applications ranging from gas sensor to solid-state electrolytes and positive electrodes for rechargeable batteries. ${ }^{1-3}$ It is an alternative photocatalyst to $\mathrm{TiO}_{2}$ for organic pollutant removal in gasoline or water via photocatalytic oxidation, e.g thiophene oxidation with loaded $\mathrm{Pt}$ and $\mathrm{RuO}_{2}$ cocatalysts. ${ }^{4} \mathrm{BiVO}_{4}$ is also a common photoanode for photoelectrochemical water splitting for several reasons. It has a band gap around $2.4 \mathrm{eV}$ absorbing light in the visible range and an appropriate valence band position for $\mathrm{O}_{2}$ evolution driving water oxidation with an onset potential of $0.3 \mathrm{~V}$ vs RHE $\left(0.9 \mathrm{~V}\right.$ vs the $\mathrm{O}_{2} / \mathrm{H}_{2} \mathrm{O}$ potential $) .{ }^{5,6}$ Its conduction band edge position is fairly negative or just located at the $\mathrm{H}_{2} / \mathrm{H}_{2} \mathrm{O}$ potential level. For that, sacrificial reagents (e.g. $\left.\mathrm{AgNO}_{3}\right)$ to improve its activity are utilized, or a small amount of external bias applied, or a hydrogen-producing photocathode is used in a tandem water splitting arrangement. ${ }^{78}$ Also, $\mathrm{BiVO}_{4}$ photoanodes do not require the use of strongly acidic or basic media to achieve an optimum photoelectrochemical performance.

$\mathrm{BiVO}_{4}$ has three crystalline phases; monoclinic clinobisvanite, scheelite-tetragonal, and zircon-tetragonal. Among the three possible phases, monoclinic shows the highest photocatalytic activity for visible-light-driven $\mathrm{O}_{2}$ evolution. ${ }^{9}$ This result attracted many researchers to mainly work on the monoclinic phase of bismuth vanadate to better understand the photocatalytic trend of this material. ${ }^{7,8}$ The photocatalytic nature of $\mathrm{BiVO}_{4}$ is not only dependent on the crystalline phases but also on the morphology controlled by different synthesis methods ${ }^{10-12}$ and on the atomic arrangement designed by proper doping techniques. ${ }^{13}$ Doping by introducing an extrinsic defect through the addition of a transition-metal cation or an anion can increase the carrier density and thus improve the conductivity for optimum photocatalytic performance. Following 
this strategy, numerous studies and research efforts have been focused on doping $\mathrm{BiVO}_{4}$ with $\mathrm{Mo}$ and $\mathrm{W}^{14-17}$

This effect has been extended as well to intrinsic doping defined as native defects. Some studies suggested that this oxide can be stoichiometric at 1 atmospheric oxygen pressure, while oxygen vacancies might be created in reducing conditions. ${ }^{18}$ This was observed by resistivity changes during a step change in the oxygen pressure. Oxygen-related defects were found to be the dominant factor for carrier conductivity. Interstitial $\mathrm{V}$ and substitutional $\mathrm{V}$ at $\mathrm{Bi}$ site are donor-like defects, and were reported for the highest film performance slightly enriched in V. ${ }^{5}$ Other defects like Bi vacancy, $\mathrm{V}$ vacancy, and substitutional $\mathrm{Bi}$ at $\mathrm{V}$ site are acceptor-like defects with p-type conductivity as indicated from the Fermi level pinning positions. For instance, Marta et al. invoked in 2015 the presence of V-vacancies from the reduction of the oxidation state of other elements. ${ }^{19}$ Recently, Bi-vacancies in a porous-like surface results in 15 -fold higher photocatalytic activity and valence band up shifting of $0.150 \mathrm{eV} .^{20}$ The electronic band gap is one of the most important factors that can be affected by the presence of such defects, leading to the creation of new sub-gap states that might act as recombination centers. ${ }^{21}$

Previous experimental works have reported band gaps in the 2.4-2.6 eV range of monoclinic $\mathrm{BiVO}_{4}$ and theoretical calculations based on density functional theory (DFT) using standard GGA exchange-correlation functional like Perdew-Burke-Emzerhof (PBE) were performed for validation attempts. ${ }^{15,22}$ However, more accurate calculations using hybrid functionals like HeydScuseria-Ernzerhof (HSE06) predicted a band gap energy around $3.0 \mathrm{eV} \cdot{ }^{23}$ This observation was surprising because semi-local GGA functionals generally strongly underestimate the band gap of semiconductors, while hybrid functionals like HSE06 are well known to give better agreement with experiment. ${ }^{24,25}$ Therefore, a deep computational investigation on the impact of possible 
intrinsic defects (such as vacancies, interstitials, and antisite defects) that might be presented experimentally on the fundamental properties of this compound was really needed to understand this discrepancy.

Here, we present a deep DFT-based computational study on key fundamental parameters of pristine and intrinsic defective monoclinic $\mathrm{BiVO}_{4}$ materials in direct correlation with photochemical water splitting application based on DFT along with the HSE06 functional and the spin-orbit-coupling (SOC) inclusion. In addition to the thermodynamic stability of the various explored intrinsic defects, we have investigated the electronic structures and optical absorption coefficient spectra for the most favorable materials. Then, we have calculated their macroscopic (optical + vibrational) dielectric tensors, their effective hole and electron mass tensors as well as their hybridization orbital characters. Finally, a candidate intrinsic defective structure was proposed to be at the origin of the poor $\mathrm{H}_{2}$ evolution performance of this material.

\section{Computational Details}

Many possible intrinsic defects in the monoclinic pristine $\mathrm{BiVO}_{4}$ (point group: $\mathrm{C}_{2 h}^{6}$, space group: $\mathrm{C} 2 / \mathrm{c}$ ) were modeled starting from the $2 \times 1 \times 2$ supercell model consisting of two-duplicated unit cells along the $a$ - and $c$-axes (16 functional units $\mathrm{Bi}_{16} \mathrm{~V}_{16} \mathrm{O}_{64}$ or 96 atoms), as shown in Figure 1. Different intrinsic defective structural configurations, namely Bi-interstitial, V-interstitial, Ointerstitial, Bi-vacancy, V-vacancy, O-vacancy, V-antisite on Bi site, Bi-antisite on V site, and V-antisite on Bi site along with additional Bi-vacancy, were studied. For Bi-, V- and O-enriched $\mathrm{BiVO}_{4}$, one neutral $\mathrm{Bi}, \mathrm{V}$ and $\mathrm{O}$ atom was inserted into the $\mathrm{Bi}_{16} \mathrm{~V}_{16} \mathrm{O}_{64}$ supercell and labeled by $\mathrm{Bi}_{(1+x)} \mathrm{VO}_{4}, \mathrm{BiV}_{(1+x)} \mathrm{O}_{4}$ and $\mathrm{BiVO}_{(4+x)}$ with $x=0.0625$. For Bi-, $\mathrm{V}$ - and O-deficient $\mathrm{BiVO}_{4}$, one neutral $\mathrm{Bi}, \mathrm{V}$ and $\mathrm{O}$ atom was removed from the $\mathrm{Bi}_{16} \mathrm{~V}_{16} \mathrm{O}_{64}$ supercell and labeled by $\left.\mathrm{Bi}_{(1-x)}\right) \mathrm{VO}_{4}$, 
$\mathrm{BiV}_{(1-x)} \mathrm{O}_{4}$ and $\mathrm{BiVO}_{(4-x)}$ with $x=0.0625$. Besides, one neutral $\mathrm{V}$ atom was substituted at on $\mathrm{Bi}$ site into the $\mathrm{Bi}_{16} \mathrm{~V}_{16} \mathrm{O}_{64}$ supercell and labeled by $\mathrm{Bi}_{(1-x)} \mathrm{V}_{(1+x)} \mathrm{O}_{4}(x=0.0625)$ as well as one neutral substitutional $\mathrm{Bi}$ at $\mathrm{V}$ site (labeled by $\mathrm{Bi}_{(1+x)} \mathrm{V}_{(1-x)} \mathrm{O}_{4}$ with $x=0.0625$ ) was considered. Moreover, three neutral $\mathrm{V}$ atoms were substituted at three $\mathrm{Bi}$ site into the $\mathrm{Bi}_{16} \mathrm{~V}_{16} \mathrm{O}_{64}$ supercell together with two additional removed neutral $\mathrm{Bi}$ atoms and labeled by $\mathrm{Bi}_{(1-5 x)} \mathrm{V}_{(1+3 x)} \mathrm{O}_{4}$ with $x=0.0625$. For each intrinsic defect type, several possible conformations were explored in order to find the most favorable structural situation. In what follows, these stoichiometry notations will used throughout the manuscript.

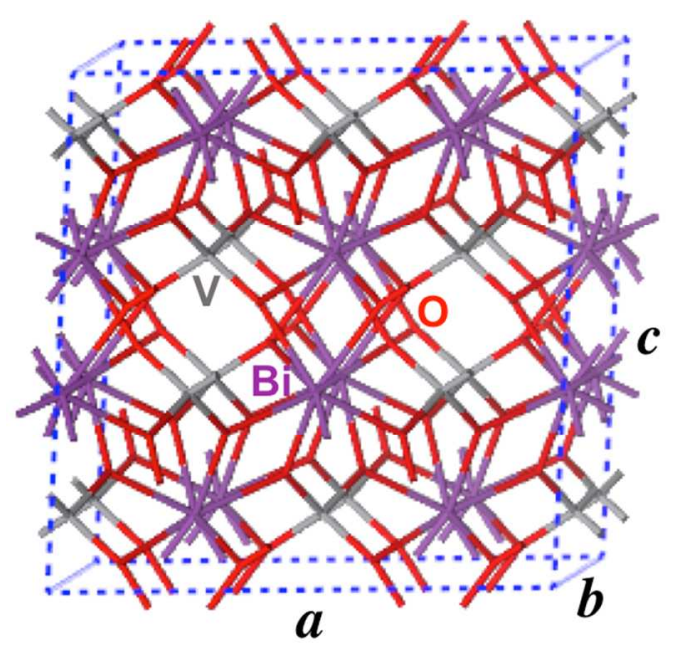

Figure 1. $2 \times 1 \times 2$ supercell model of monoclinic $\mathrm{BiVO}_{4}$ crystalline phase. $\mathrm{Bi}, \mathrm{V}$, and $\mathrm{O}$ are shown in purple, gray, and red.

All of generated configurations were fully relaxed using spin-polarized density functional theory (DFT) with the plane wave (PW) method as implemented in VASP 5.4 quantum simulation code. ${ }^{26-28}$ The Perdew-Burke-Ernzerhof (PBE) exchange-correlation functional, ${ }^{29}$ the projector augmented-wave (PAW) method, ${ }^{30}$ a cutoff kinetic energy of $400 \mathrm{eV}$ for electron wave basis functions, and a $3 \times 3 \times 3$ Monkhorst-Pack $k$-point grid $^{31}$ for sampling the Brillouin zone, were employed. Benchmark tests with increased cutoff energy up to $500 \mathrm{eV}$ or including or by 
including $\mathrm{Bi}$ 5d-electrons on $\mathrm{Bi}$ revealed that the structural parameters such as lattice lengths/angles and bond lengths were successfully converged at $400 \mathrm{eV}$. Because the magnetization is dependent on the intrinsic defect type, several multiplicities of spin were systematically checked for each intrinsic defective conformation to identify the most favorable spin configuration. The geometry was re-optimized for each magnetic moment. The valence electron configurations taken into account in PAW potentials are $6 s^{2} 6 p^{3}$ for $\mathrm{Bi}, 3 \mathrm{p}^{6} 3 \mathrm{~d}^{4} 4 \mathrm{~s}^{1}$ for $\mathrm{V}$, and $2 s^{2} 2 p^{4}$ for $\mathrm{O}$. The convergence criterion for the self-consistent-field (SCF) cycles for energy change was set to $10-6 \mathrm{eV}$ per supercell. The cell parameters and atomic positions were fully optimized until the three principal residual Hellmann-Feynman force components on each atom were below $0.01 \mathrm{eV} / \AA$.

Relative stability of the various explored intrinsic defective $\mathrm{BiVO}_{4}$ structures with respect to the pristine one was investigated by computing the defect formation energies under the experimental conditions, following the method that has been successfully applied to investigate the thermodynamic stability of various defective materials used in photocatalysis or photoelectrochemitry, and was in line with the experimental observations. ${ }^{32-34}$ Benchmark tests with increased cutoff energy up to $500 \mathrm{eV}$ or including $\mathrm{Bi} 5 \mathrm{~d}$-electrons on $\mathrm{Bi}$ revealed minor impact on the formation energy.

The electronic structure properties of monoclinic pristine and the most relevant intrinsic defective $\mathrm{BiVO}_{4}$ crystals were investigated by computing their density of states (DOS) together with the partial charge density maps corresponding to their band edge and mid-gap electronic states in terms of orbital contribution types of each element in the cell. The calculations were carried out using the Heyd-Scuseria-Ernzerhof (HSE06) functional, ${ }^{35,36}$ which is well documented to give more accurate band gap values of semiconductors than standard GGA 
functionals. $^{24,37-39}$ The spin-orbit-coupling (SOC) effect on the band gap was also taken into account by including relativistic effects on $\mathrm{Bi}$. The $5 \mathrm{~d}$-electrons on $\mathrm{Bi}$ were not considered as valence electrons in Bi because they would not change the band gap and DOS characteristics as recently reported in the literature. ${ }^{40}$ An expanded cutoff energy up to $500 \mathrm{eV}$ for basis functions and an increased $5 \times 5 \times 5$ Monkhorst-Pack $k$-point grid for the Brillouin zone were used to ensure sufficient convergence of the band gap values of the materials.

The optical absorption spectra of pristine and the most relevant intrinsic defective $\mathrm{BiVO}_{4}$ crystals were computed by means of the sum over states (SOS) method implemented in VASP with HSE06. To get the fraction of absorbed light in the crystals, we computed in terms of upcoming incident photon wavelength the absorption coefficient through the extinction coefficient that is dependent on the two parts of the complex dielectric function (imaginary and real). The imaginary part was computed by taking into account all possible excitations from filled to empty orbitals weighted by the momentum matrix elements describing the transition probability. The real part was obtained from the imaginary part by means of the Kramers-Kronig transformation. This method has been successfully applied to investigate the optical absorption properties of various materials (including intrinsic or extrinsic doping) used in solar energy conversion applications, and has shown results in line with the experimental measurements. ${ }^{37,38}$

\section{Results and Discussion}

\section{III.1. Structures and Stabilities}

Lowest-energy structures for the various explored intrinsic defects in $\mathrm{BiVO}_{4}$ including $\mathrm{Bi}$ - and $\mathrm{V}$-antisites, Bi-, $\mathrm{V}-$, and $\mathrm{O}$-interstitials as well as the possible formation of $\mathrm{Bi}-, \mathrm{V}-$ and $\mathrm{O}$ vacancies, are displayed in Figure 2. Those structures were fully relaxed using DFT within the 
PBE functional since it yields accurate bonds and lattice parameters in computationally cheap method. The calculated lattice parameters of the relaxed structures are reported in Table 1. These values are found to be in good agreement with the experimental lattice constants, with deviation of less than $1 \%{ }^{41}$

(a)

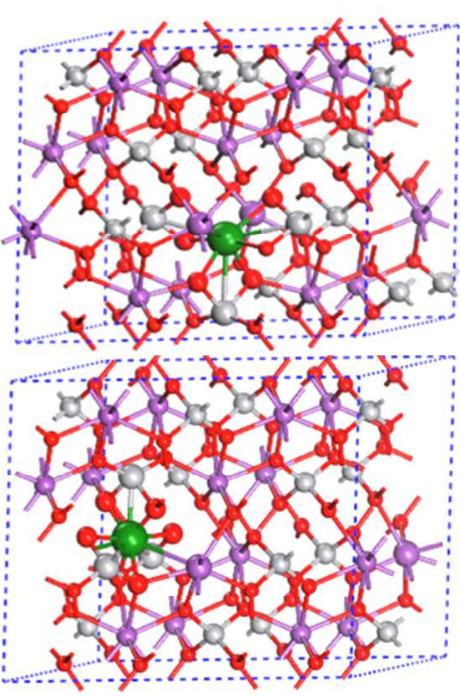

(c)

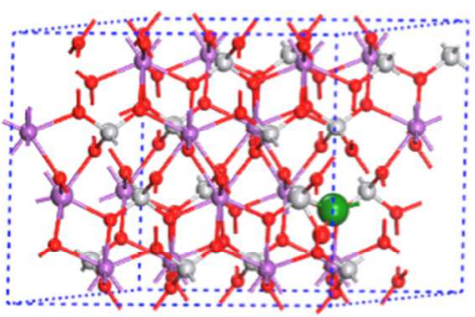

(d)

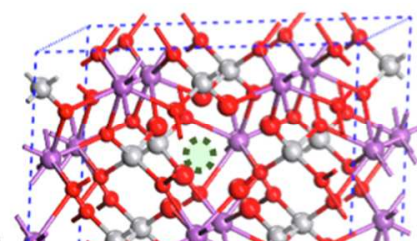

(e)

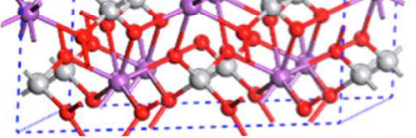

(f)
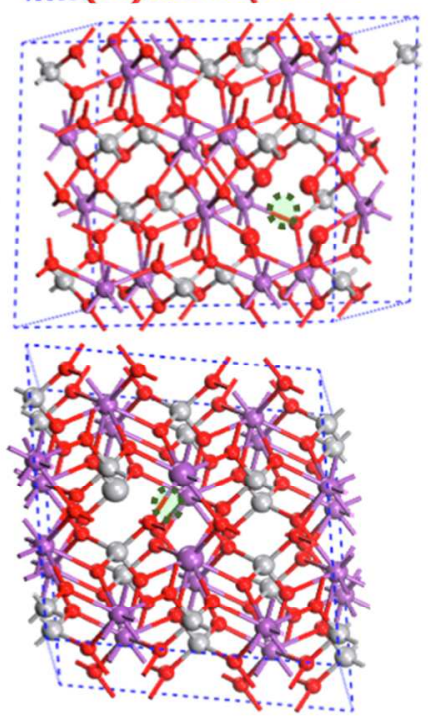

(g)

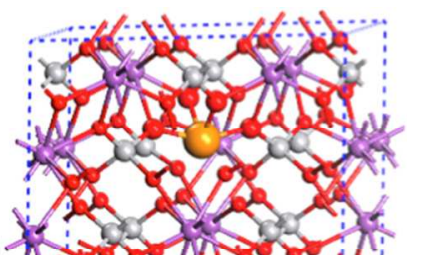

(h)
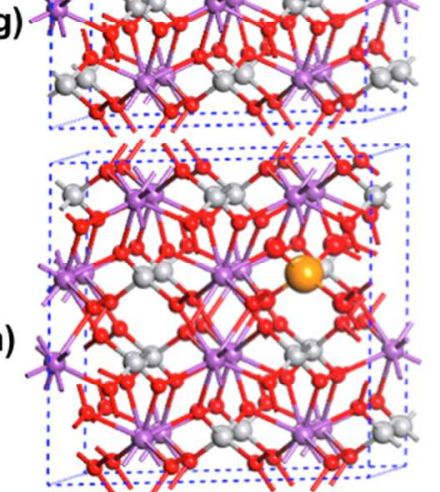

(i)

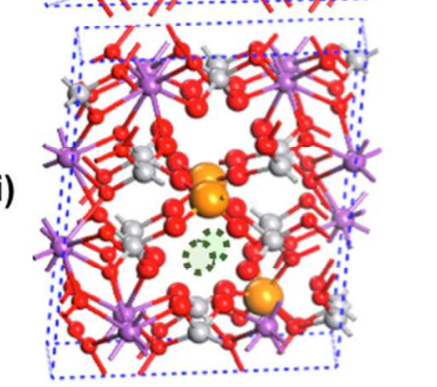

Figure 2. Lowest-energy configurations of the various intrinsic defects in $\mathrm{BiVO}_{4}$ : (a) $\mathrm{Bi}_{(1+x)} \mathrm{VO}_{4}$, (b) $\mathrm{BiV}_{(1+x)} \mathrm{O}_{4}$, (c) $\mathrm{BiVO}_{(4+x)}$, (d) $\mathrm{Bi}_{(1-x)} \mathrm{VO}_{4}$, (e) $\mathrm{BiV}_{(1-x)} \mathrm{O}_{4}$, (f) $\mathrm{BiVO}_{(4-x)}$, (g) $\mathrm{Bi}_{(1-x)} \mathrm{V}_{(1+x)} \mathrm{O}_{4}$, (h) $\mathrm{Bi}_{(1+x)} \mathrm{V}_{(1-x)} \mathrm{O}_{4}$, and (i) $\mathrm{Bi}_{(1-5 x)} \mathrm{V}_{(1+3 x)} \mathrm{O}_{4}$ (with $x=0.0625$ ). $\mathrm{Bi}$ is shown in purple, $\mathrm{V}$ in gray, and $\mathrm{O}$ in red. Interstitial/antisite atoms are shown in green and vacant sites are shown in green circle.

Table 1. Optimized Lattice Parameters and Calculated Formation Energies at $\mathrm{T}=723 \mathrm{~K}$ in $\mathrm{O}_{2}$ Atmosphere for the Various Explored Intrinsic Defective $\mathrm{BiVO}_{4}$ Structures Reported in Figure 2.

\begin{tabular}{ccccccccc}
\hline \multirow{2}{*}{ material } & \multirow{2}{*}{$\begin{array}{c}\text { formation } \\
\text { structure }\end{array}$} & \multicolumn{6}{c}{ lattice parameters (lengths in $\AA$; angles in ${ }^{\circ}$ ) } \\
\cline { 4 - 9 } & & & & \multicolumn{7}{c}{$c$} & $b$ & $c$ & $\alpha$ & $\beta$ & $\gamma$ \\
\hline $\mathrm{BiVO}_{4}$ & $\mathrm{Expt}^{41}$ & - & 7.25 & 11.70 & 5.09 & 90 & 134.2 & 90 \\
$\mathrm{BiVO}_{4}$ & $(\mathrm{DFT})$ & - & 7.23 & 11.56 & 5.10 & 90 & 134.8 & 90
\end{tabular}




$\begin{array}{ccccccccc}\mathrm{Bi}_{(1+x)} \mathrm{VO}_{4} & (2 \mathrm{a}) & 0.39 & 7.27 & 11.62 & 5.13 & 90 & 134.3 & 89.9 \\ \mathrm{BiV}_{(1+x)} \mathrm{O}_{4} & (2 \mathrm{~b}) & 0.04 & 7.27 & 11.55 & 5.12 & 90 & 134.8 & 89.9 \\ \mathrm{BiVO}_{(4+x)} & (2 \mathrm{c}) & 0.20 & 7.25 & 11.57 & 5.12 & 90 & 135.1 & 89.9 \\ \mathrm{Bi}_{(1-x)} \mathrm{VO}_{4} & (2 \mathrm{~d}) & 0.46 & 7.21 & 11.50 & 5.10 & 90 & 134.9 & 90 \\ \mathrm{BiV}_{(1-x)} \mathrm{O}_{4} & (2 \mathrm{e}) & 0.96 & 7.23 & 11.48 & 5.11 & 90 & 134.9 & 90 \\ \mathrm{BiVO}_{(4-x)} & (2 \mathrm{f}) & 0.27 & 7.21 & 11.54 & 5.10 & 89.8 & 134.8 & 90.2 \\ \mathrm{Bi}_{(1-x)} \mathrm{V}_{(1+x)} \mathrm{O}_{4} & (2 \mathrm{~g}) & 0.02 & 7.22 & 11.55 & 5.08 & 89.9 & 135 & 90 \\ \mathrm{Bi}_{(1+x)} \mathrm{V}_{(1-x)} \mathrm{O}_{4} & (2 \mathrm{~h}) & 0.44 & 7.31 & 11.54 & 5.17 & 90.1 & 135.3 & 90 \\ \mathrm{Bi}_{(1-5 x)} \mathrm{V}_{(1+3 x)} \mathrm{O}_{4} & (2 \mathrm{i}) & 0.13 & 7.18 & 11.72 & 5.02 & 89.8 & 135.4 & 90\end{array}$

Among the various explored intrinsic defective structures, several ones showed very low relative formation energies to the perfect structure, as listed in Table 1. Considering the structures with less than $0.2 \mathrm{eV}$ difference are stable, V-rich structures (containing both Vinterstitials and $\mathrm{V}$-antisites on $\mathrm{Bi}$ without/with the formation of additional Bi-vacancies) and $\mathrm{O}$ rich structures (containing O-interstitials) were found the most stable ones. In contrast, Bi-rich structures (containing both Bi-interstitials and Bi-antisites on V), Bi-deficient structures (containing Bi-vacancies), V-deficient structures (containing V-vacancies), and O-deficient structure (containing O-vacancies) were considered as metastable. Though, both groups of structures were further electronically investigated, and this will be the matter of the next section.

\subsection{Electronic Structure Properties}

The calculated energy bands of pristine monoclinic $\mathrm{BiVO}_{4}$ along the high symmetry directions of the Brillouin zone are displayed in Figure S1 (Supporting Information). The selected $k$-points of (L-M-A- $\Gamma-Z)$ were employed in previous studies. ${ }^{42}$ The band gap type was found to be indirect in line with previous reports. ${ }^{43,44}$ Figure S2 (in the Supporting Information) presents the 
impact of spin-orbit coupling (SOC) inclusion on the electronic density of states (DOS) calculation of pristine $\mathrm{BiVO}_{4}$ at the PBE level of functional. The band gap was reduced by 0.2 $\mathrm{eV}$ due to the strong relativistic effect of bismuth atoms. Though, our PBE calculated band gap value of $1.9 \mathrm{eV}$ without SOC was close to the previous theoretical studies. The same SOC effect on the band gap was even observed through the DOS calculations at the HSE06 level of functional, as displayed in Figure 3. The obtained band gap is $3.0 \mathrm{eV}$ without SOC inclusion, which reproduces previously reported band gaps. ${ }^{23,43}$ The main contribution in the conduction band comes from the V $3 \mathrm{~d}$ orbitals. However, the valence band mainly originates from $\mathrm{O} 2 \mathrm{p}$ orbitals with minor $\mathrm{Bi} 6 \mathrm{~s}$ orbitals contribution, in line with experimental photoemission results. ${ }^{8,45}$ Almost the same types of orbital contributions were also found in all other defective structures.

(a)

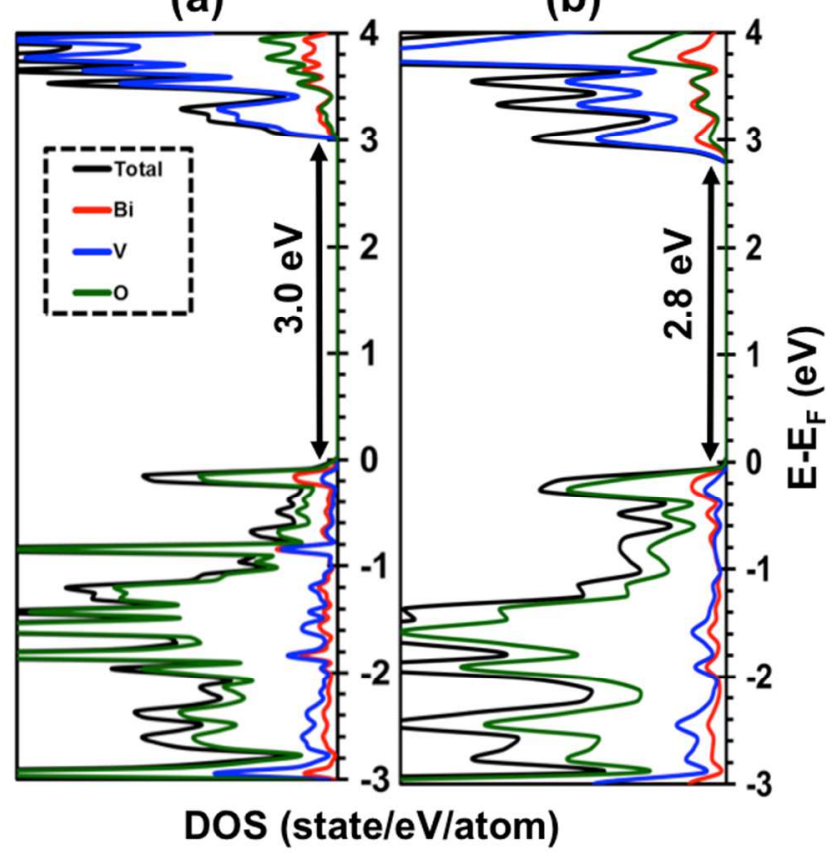

Figure 3. DOSs obtained with HSE06 for pristine $\mathrm{BiVO}_{4}$ : (a) without spin-orbit coupling (SOC) effect and (b) including SOC. DOSs projected on Bi 6s, V 3d, and O 2 p orbitals are shown in red, blue, and green colors. 

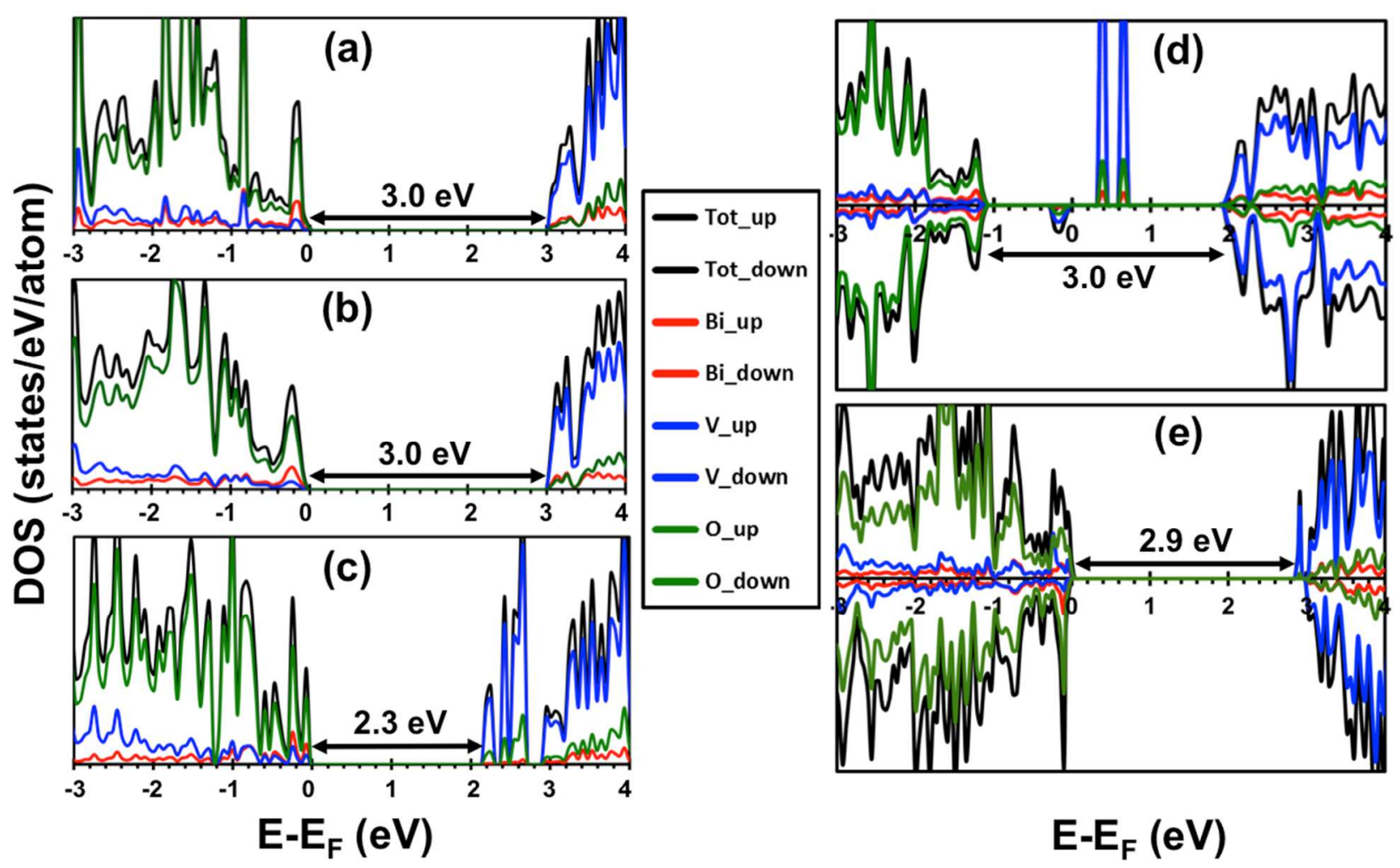

Figure 4. DOSs obtained with HSE06 for pristine and the most favorable intrinsic defective $\mathrm{BiVO}_{4}$ materials in their most stable spin state configurations: (a) $\mathrm{BiVO}_{4}$, (b) $\mathrm{BiVO}_{(4+x)}$, (c) $\mathrm{Bi}_{(1-}$ ${ }_{5 x} \mathrm{~V}_{(1+3 x)} \mathrm{O}_{4}$, (d) $\mathrm{BiV}_{(1+x)} \mathrm{O}_{4}$, and (e) $\mathrm{Bi}_{(1-x)} \mathrm{V}_{(1+x)} \mathrm{O}_{4}$ (with $x=0.0625$ ). Singlet spins for (a)-(c), quartet for (d), and doublet for (e). DOSs projected on Bi 6s, V 3d, and O 2p orbitals are shown in red, blue, and green colors. The Fermi level $\left(\mathrm{E}_{\mathrm{F}}\right)$ is set at $0 \mathrm{eV}$.

The structure of pristine $\mathrm{BiVO}_{4}$ is neutral and has balanced number of electrons, whereas introducing defects can change the number of released electron/holes and the charge carrier density. For that, several possible multiplicities of spin were checked for each intrinsic defective structure to find the lowest energy structure with the optimal spin configuration. The defect usually can create new empty or occupied states in the gap. Our most stable defective structures $\mathrm{V}$-interstitial and $\mathrm{V}$-antisite on $\mathrm{Bi}$, are donor-like defects and have been reported experimentally as the highest film performance slightly enriched in $V^{5}$ Figure 4 displays the DOS plots for pristine and the most favorable intrinsic defective $\mathrm{BiVO}_{4}$ structures using HSE06. Intrinsic band gaps were found for all of them (Figure 4a-c, e), except for V-interstitial which revealed new 
sharp mid-gap occupied and empty states that might act as traps for electron/hole recombination (Figure 4d). Among the most favorable structures, only that corresponding to $\mathrm{Bi}_{(1-5 x)} \mathrm{V}_{(1+3 x)} \mathrm{O}_{4}$ (with $x=0.0625$ ) has given a reduced band gap with a value around $2.3 \mathrm{eV}$ (Figure 4c), which is very close to the experimentally reported ones. ${ }^{46,47}$
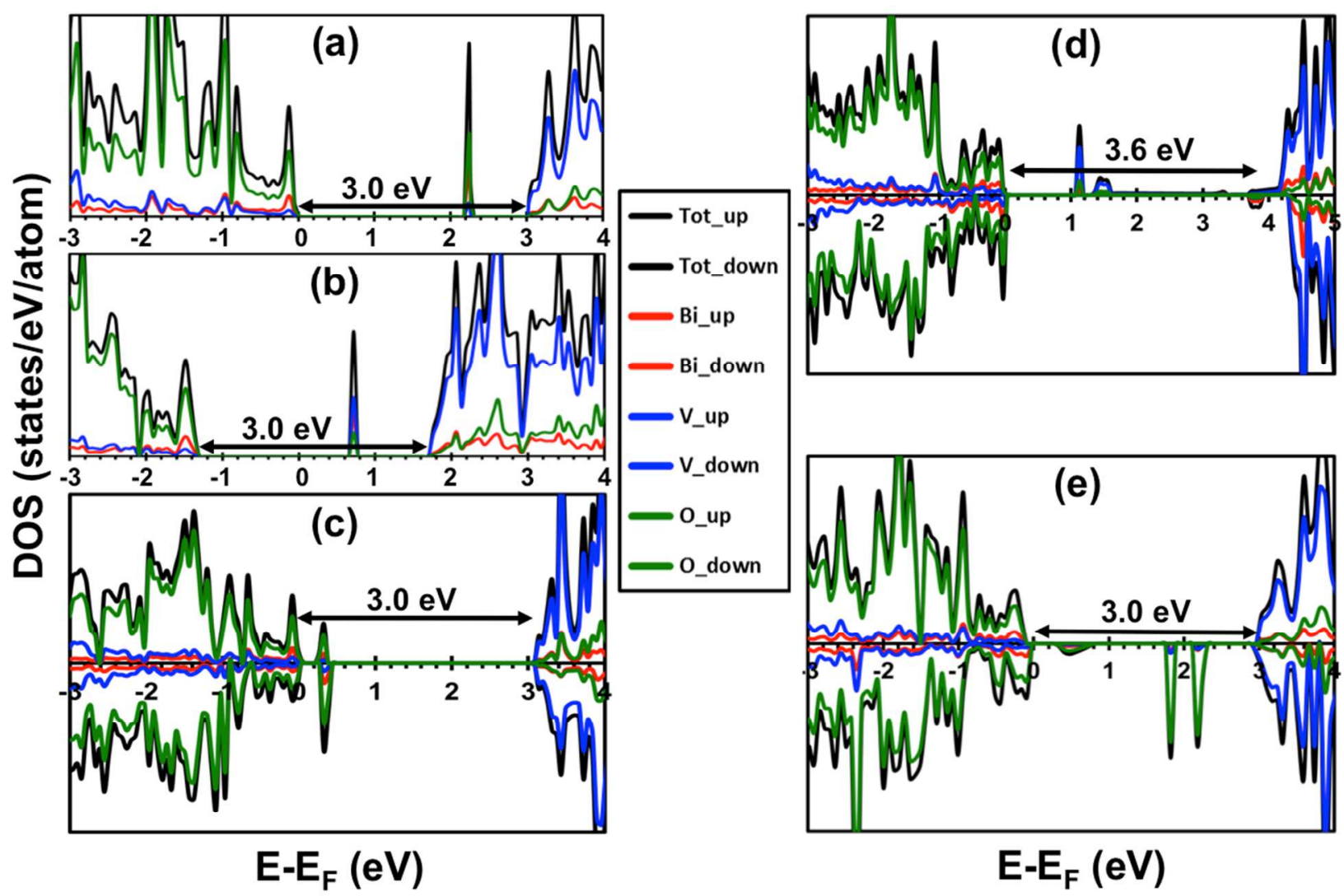

Figure 5. DOSs obtained with HSE06 for the metastable intrinsic defective $\mathrm{BiVO}_{4}$ materials in their most stable spin state configurations: (a) $\mathrm{Bi}_{(1+x)} \mathrm{V}_{(1-x)} \mathrm{O}_{4}$, (b) $\mathrm{BiVO}_{(4-x)}$, (c) $\mathrm{BiV}_{(1-x)} \mathrm{O}_{4}$, (d) $\mathrm{Bi}_{(1-x)} \mathrm{VO}_{4}$, and (e) $\mathrm{Bi}_{(1+x)} \mathrm{VO}_{4}$ (with $x=0.0625$ ). Singlet spins for (a) and (b), doublet for (c), quartet for (d), and sextet for (e). DOSs projected on Bi 6s, V $3 \mathrm{~d}$, and $\mathrm{O} 2 \mathrm{p}$ orbitals are shown in red, blue, and green colors. The Fermi level $\left(\mathrm{E}_{\mathrm{F}}\right)$ is set at $0 \mathrm{eV}$.

It is known that the creation of fully delocalized doping levels on top of the original valence band maximum (VBM) or just below the original conduction minimum (CBM) could narrow the band gap and help for avoiding the inefficient electron/hole recombination. For that aim, the metastable structures were also examined using HSE06 and their DOS plots are shown in Figure 
5. All of them behaved as acceptor-like defects revealing new empty states located either in the middle of the original band gap (Figure 5d) or closer to the CBM (Figure 5a, b, e) or closer to the VBM (Figure 5c). The sharp or tight character of these states comes from their localized nature around the corresponding defects, and this might act as traps for electron/hole recombination. As a consequence, only the stoichiometric $\mathrm{BiVO}_{4}$ and the intrinsic defective material containing Vantisites on $\mathrm{Bi}$ along with the formation of additional Bi-vacancies (labeled by $\mathrm{Bi}_{(1-5 x)} \mathrm{V}_{(1+3 x)} \mathrm{O}_{4}$ ) were considered for further investigations on the optoelectronic properties.

It is experimentally reported that $\mathrm{BiVO}_{4}$ has different $\mathrm{Bi}-\mathrm{O}$ and $\mathrm{V}-\mathrm{O}$ bond length types signifying that the two $\mathrm{VO}_{4}$ tetrahedra and $\mathrm{BiO}_{8}$ polyhedra are slightly distorted. ${ }^{47}$ This is the case for all the optimized intrinsic defective $\mathrm{BiVO}_{4}$ structures displayed in Figure 2, but not for the perfect one as shown in Table 2. In the case of the intrinsic defective $\mathrm{Bi}_{(1-5 x)} \mathrm{V}_{(1+3 x)} \mathrm{O}_{4}$ structure, the coordination environment of $\mathrm{Bi}$ clearly showed a distorted $\mathrm{O}$ polyhedral environment with the nearest neighbor distances ranging from 2.27 to $2.59 \AA$. One of the substituted vanadium species also had more distortion in the tetrahedral environment. It is believed that the former distortion may improve the lone pair effect of Bi 6 s orbitals and upward shift the original VBM to give the reduced band gap.

Table 2. Bond Lengths of Pristine $\mathrm{BiVO}_{4}$ and the Intrinsic Defective $\mathrm{Bi}_{(1-5 x)} \mathrm{V}_{(1+3 x)} \mathrm{O}_{4}(x=$ 0.0625) Materials. $\mathrm{V}^{*}$ Represents one of the Vanadium Antisite Atoms on Bi. Bi* Represents Bismuth Atoms Located Near the Defect Site.

\begin{tabular}{cccccc}
\hline \multirow{2}{*}{ material } & \multicolumn{5}{c}{ bond lengths $(\AA)$} \\
\cline { 2 - 6 } & $\mathrm{V}-\mathrm{O}$ & $\mathrm{Bi}-\mathrm{O}$ & $\mathrm{Bi}-\mathrm{O}$ & $\mathrm{V} *-\mathrm{O}$ & $\mathrm{Bi}^{*}-\mathrm{O}$ \\
\hline $\mathrm{BiVO}_{4}$ & 1.73 & 2.41 & - & - & - \\
& & 2.45 & & \\
\hline
\end{tabular}




\begin{tabular}{cccccc}
\hline $\mathrm{Bi}_{(1-}$ & 2.32 & 1.73 & 1.75 & 2.27 \\
${ }_{5 x)} \mathrm{V}_{(1+3 x)} \mathrm{O}_{4}$ & & 2.35 & 1.75 & 1.78 & 2.41 \\
& 1.67 & 2.38 & & 1.69 & 2.43 \\
& 1.73 & 2.43 & 1.96 & 2.49 \\
& 2.47 & & 2.11 & 2.54 \\
& 2.59 & & &
\end{tabular}

\subsection{Optical Absorption and Dielectric Properties}

The optical absorption coefficient edge for the intrinsic defective $\mathrm{Bi}_{(1-5 x)} \mathrm{V}_{(1+3 x)} \mathrm{O}_{4}(x=0.0625)$ material revealed a red shift by $140 \mathrm{~nm}$ compared to the pristine $\mathrm{BiVO}_{4}$ (Figure 6). The former has an absorbance onset at around $540 \mathrm{~nm}$ leading to visible-light absorption, while the stoichiometric $\mathrm{BiVO}_{4}$ has an onset at around $410 \mathrm{~nm}$ that is at the border between both UV and visible range.

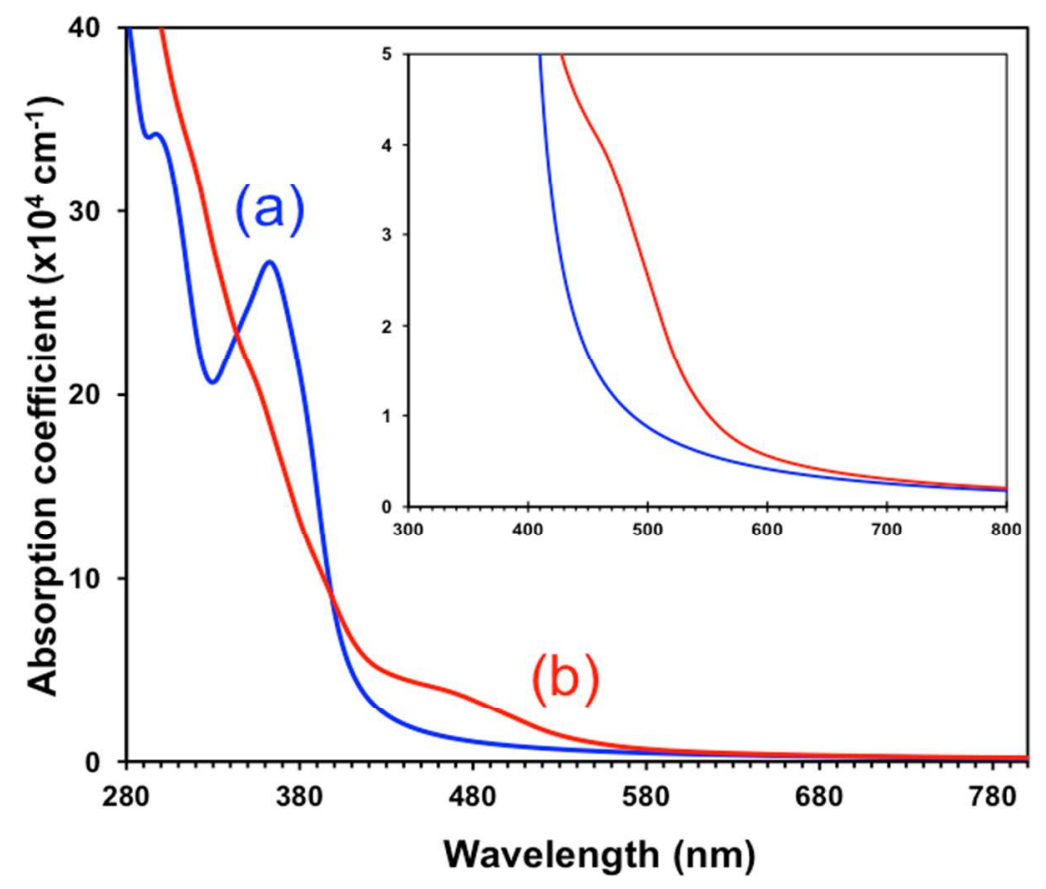

Figure 6. Simulated UV-visible optical absorption edges with $\mathrm{HSE} 06$ for (a) pristine $\mathrm{BiVO}_{4}$ and (b) the intrinsic defective $\mathrm{Bi}_{(1-5 x)} \mathrm{V}_{(1+3 x)} \mathrm{O}_{4}(x=0.0625)$ materials. 
The averaged real and imaginary parts of the incident light wavelength-dependent dielectric function in the three main polarization directions were used to simulate UV-visible absorption edges of the two materials. The real part $\left(\varepsilon_{1}\right)$ is responsible for slowing down the speed of light in the material by dispersion, whereas the imaginary part $\left(\varepsilon_{2}\right)$ is responsible for the absorption energy from the applied external electric field due to the internal induced dipole motion. The dependence of $\varepsilon_{1}$ and $\varepsilon_{2}$ values on the incident photon energy is, respectively, shown in Figure 7 along the three principal crystallographic directions. Due to the monoclinic symmetry of pristine structure, the dielectric tensor for both parts was anisotropic with $\varepsilon_{x x}=\varepsilon_{z z} \neq \varepsilon_{y y}$. In contrast, $\varepsilon_{x x}$ was slightly different from $\varepsilon_{z z}$ in the case of intrinsic defective $\mathrm{Bi}_{(1-5 x)} \mathrm{V}_{(1+3 x)} \mathrm{O}_{4}(x=0.0625)$ structure because the corresponding lattice parameters were slightly changed. From Figure 7a,b, the band gap can be confirmed from the tangent adjacent to the linear region of the absorption intersecting with the photon energy at the band gap value. This approach is called the "tangent approach" to determine the optical band gap. The real part of the dielectric function in Figure $7 \mathrm{c}, \mathrm{d}$ was used to drive the electronic or optical contribution of the macroscopic dielectric constant at the photon energy corresponding to the HSE06 band gap. 

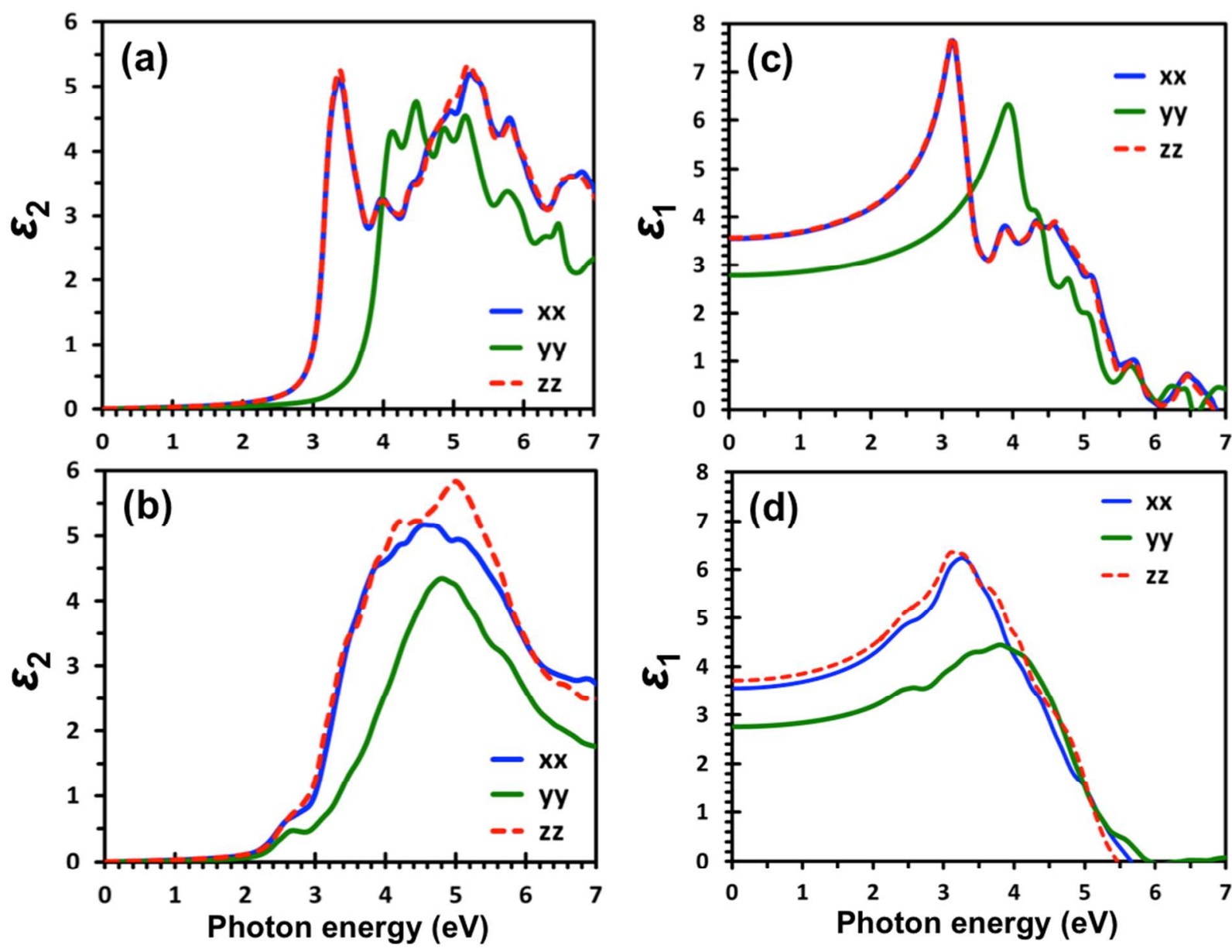

Figure 7. The imaginary (on the left) and real (on the right) parts of the dielectric function obtained by HSE06 calculations along the three principal directions for (a), (c) pristine $\mathrm{BiVO}_{4}$ and (b), (d) the intrinsic defective $\mathrm{Bi}_{(1-5 x)} \mathrm{V}_{(1+3 x)} \mathrm{O}_{4}(x=0.0625)$ materials.

The macroscopic dielectric constant $\left(\varepsilon_{\mathrm{r}}\right)$ describes the tendency of atomic charges in the material to get distorted upon applying external electric field. It has an optical contribution $\left(\varepsilon_{\infty}\right)$ that includes local field effects and describes the relaxation of crystalline orbitals. There is also a vibrational contribution $\left(\varepsilon_{\mathrm{vib}}\right)$, which involves ionic motion and can be obtained from phonon frequencies calculation by means of DFPT. Here, the two contributions were examined for pristine $\mathrm{BiVO}_{4}$ and the intrinsic defective $\mathrm{Bi}_{(1-5 x)} \mathrm{V}_{(1+3 x)} \mathrm{O}_{4}(x=0.0625)$ material, and the calculated values are given in Table 3. The averaged macroscopic dielectric constant values for 
pristine and the intrinsic defective materials were 38.3 and 41.2, respectively. Those values are close to the experimentally reported one of $52,,^{48}$ and also very close to that obtained of 45.7 for anatase $\mathrm{TiO}_{2} \cdot{ }^{49}$ Here, let us simply remind that several studies mentioned that dielectric constant value greater than 10 is sufficient for good exciton dissociation to free carriers. ${ }^{50}$ This confirms the good conductivity of bismuth vanadate, which was even enhanced by the presence of this type intrinsic defect associated with $\mathrm{V}$-antisites on Bi together with the formation of additional Bi-vacancies.

Table 3. Components Along the Three Main Directions of Electronic $\left(\varepsilon_{\infty}\right)$, Ionic $\left(\varepsilon_{\mathrm{vib}}\right)$, and Macroscopic $\left(\varepsilon_{\mathrm{r}}\right)$ Dielectric Constants for Pristine $\mathrm{BiVO}_{4}$ and the Intrinsic Defective $\mathrm{Bi}_{(1-}$ ${ }_{5 x} \mathrm{~V}_{(1+3 x)} \mathrm{O}_{4}(x=0.0625)$ Materials Obtained for $\varepsilon_{\mathrm{vib}}$ and $\varepsilon_{\infty}$ with PBE and HSE06, Respectively.

\begin{tabular}{ccccc}
\hline material & direction & $\varepsilon_{\infty}$ & $\varepsilon_{\text {vib }}$ & $\varepsilon_{\mathrm{r}}$ \\
\hline $\mathrm{BiVO}_{4}$ & $x x$ & 6.8 & 30.1 & 37.8 \\
& $y y$ & 3.8 & 32.3 & 38.8 \\
& $z z$ & 6.8 & 30.7 & 38.5 \\
\hline $\mathrm{Bi}_{(1-}$ & $x x$ & 4.6 & 27.8 & 35.3 \\
$5 x \mathrm{~V}_{(1+3 x)} \mathrm{O}_{4}$ & $y y$ & 3.4 & 24.3 & 30.3 \\
& $z z$ & 4.8 & 50.2 & 58.0
\end{tabular}

\subsection{Charge Carrier Transport Properties}

Although $\mathrm{BiVO}_{4}$ has been invoked as one of the most active photocatalysts for water oxidation driven by visible light, powder samples have been invoked to yield relatively poor photo(electro)chemical performance for $\mathrm{H}_{2}$ evolution. The reasons behind that may come from slow $\mathrm{H}_{2}$ evolution kinetics, poor electron transport from the bulk to its surface to reduce $\mathrm{H}^{+}$ions besides the electron-hole recombination in bulk and surface. ${ }^{8}$ 
$\mathrm{BiVO}_{4}$ has been reported to have a very low electron mobility that is expected to limit its overall photocatalytic performance particularly the $\mathrm{H}_{2}$ evolution side. ${ }^{51}$ The weakly dispersive valence band and conduction band in the band structure plot give an indication of the relatively localized charge carriers, hence the low mobility. As reported in Table 4, our computed effective electron masses for the intrinsic defective $\mathrm{Bi}_{(1-5 x)} \mathrm{V}_{(1+3 x)} \mathrm{O}_{4}(x=0.0625)$ material revealed much larger values than those obtained for the holes along the three crystallographic directions, confirming the very low electron mobility found in the prepared samples. In contrast, the values obtained for the effective hole masses are very similar in the three principal directions. Our smallest effective electron mass was $0.24 m_{0}$ for the pristine material in the [001] direction, while a much larger value of $4.37 m_{0}$ for the intrinsic defective $\mathrm{Bi}_{(1-5 x)} \mathrm{V}_{(1+3 x)} \mathrm{O}_{4}(x=0.0625)$ material in the [100] direction, which is even much larger than that obtained of $m_{\mathrm{e}}{ }^{*} \sim 1 m_{0}$ for anatase $\mathrm{TiO}_{2}{ }^{52,53}$ The smallest effective hole mass was $0.31 m_{0}$ for the pristine material in the [001] direction and a similar value of $0.46 m_{0}$ was obtained for the intrinsic defective $\mathrm{Bi}_{(1-5 x)} \mathrm{V}_{(1+3 x)} \mathrm{O}_{4}(x$ $=0.0625)$ material in the [001] direction. Those values are smaller than that that obtained of $m_{\mathrm{h}}{ }^{*} \sim 8 m_{0}$ for anatase $\mathrm{TiO}_{2} \cdot{ }^{54}$ To improve the photogenerated charge separation in $\mathrm{BiVO}_{4}$, a heterojunction with other semiconductors like $\mathrm{TiO}_{2}{ }^{55}$ and $\mathrm{ZnO}^{56}$ has proven experimentally to be a successful technique.

Table 4. Effective Hole $\left(m_{\mathrm{h}}{ }^{*} / m_{0}\right)$ and Electron $\left(m_{\mathrm{e}}{ }^{*} / m_{0}\right)$ Masses of Pristine $\mathrm{BiVO}_{4}$ and the Intrinsic Defective $\mathrm{Bi}_{(1-5 x)} \mathrm{V}_{(1+3 x)} \mathrm{O}_{4}(x=0.0625)$ Materials Along the Three Principal Directions as Obtained with PBE. $m_{0}$ is the Free Mass of Electron.

\begin{tabular}{cccc}
\hline material & direction & $m_{\mathrm{h}} * / m_{0}$ & $m_{\mathrm{e}} * / m_{0}$ \\
\hline $\mathrm{BiVO}_{4}$ & {$[100]$} & 2.86 & 3.57 \\
& {$[010]$} & 0.51 & 0.50 \\
& {$[001]$} & 0.31 & 0.24 \\
\hline
\end{tabular}




\begin{tabular}{cccc}
\hline $\mathrm{Bi}_{(1-}$ & {$[100]$} & 3.23 & 4.37 \\
$\mathrm{~V}_{(1+3 x)} \mathrm{O}_{4}$ & {$[010]$} & 0.65 & 8.54 \\
& {$[001]$} & 0.46 & 5.85
\end{tabular}

(a)

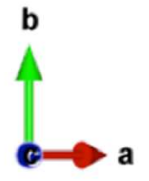

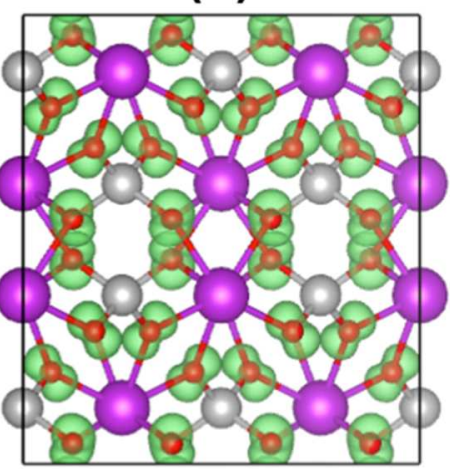

(b)
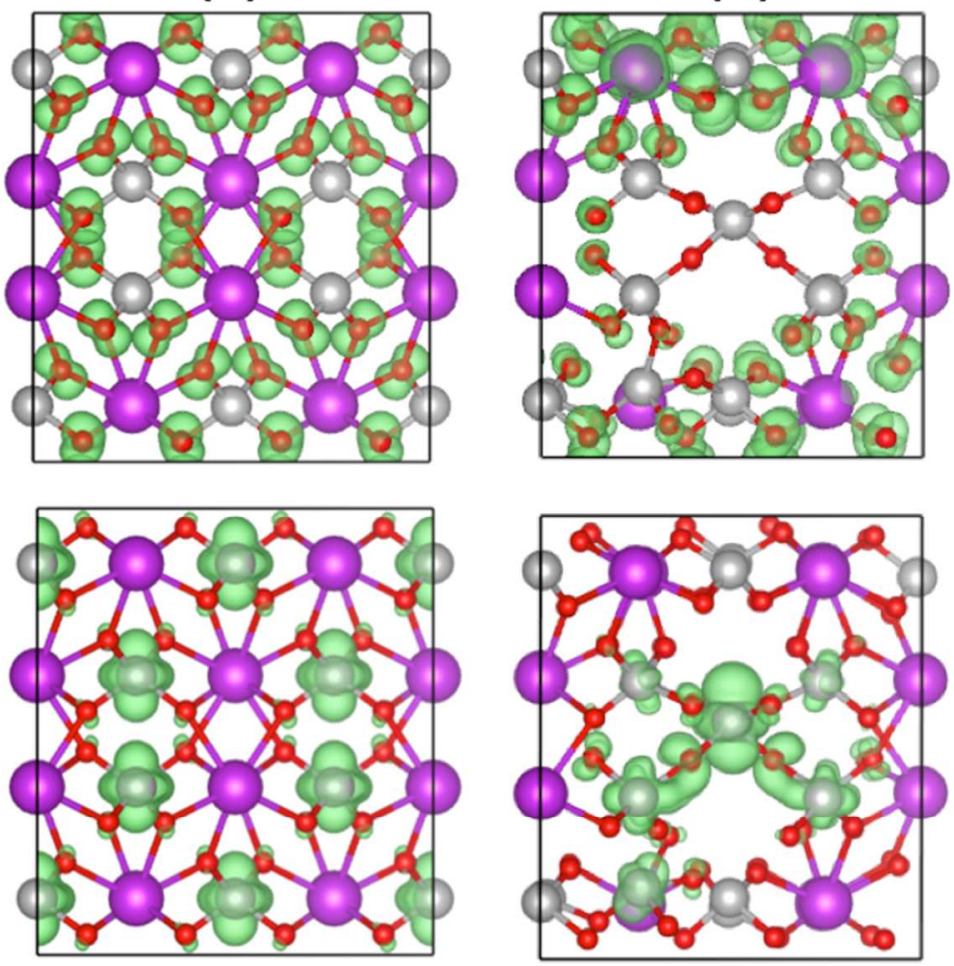

Figure 8. VBM (top) and CBM (bottom) partial charge densities obtained with HSE06 for (a) pristine $\mathrm{BiVO}_{4}$ and (b) the intrinsic defective $\mathrm{Bi}_{(1-5 x)} \mathrm{V}_{(1+3 x)} \mathrm{O}_{4}(x=0.0625)$ materials. Isovalue is 0.003 a.u.

The partial charge density maps in Figure 8 show similar properties to those obtained through the effective masses calculation. For the pristine $\mathrm{BiVO}_{4}$, both the VBM and CBM partial charge densities are delocalized throughout the structure layers promoting good migration of holes and electrons to surface. The VBM charge density is mainly made by filled $\mathrm{O} 2 \mathrm{p}$ orbitals, whereas the CBM charge density is formed by empty V 3d orbitals. This reflects the projected density of states results mentioned in the previous section. For the intrinsic defective $\mathrm{Bi}_{(1-5 x)} \mathrm{V}_{(1+3 x)} \mathrm{O}_{4}(x=$ 
0.0625 ) structure, the VBM partial charge density is delocalized on all O 2p orbitals around the defective sites and throughout the crystal lattice, while the CBM partial charge density was strongly localized only on the d-orbitals of the newly substituted $\mathrm{V}$ at $\mathrm{Bi}$ sites. This strong localization together with the absence of any overlaps with the neighboring Bi $6 \mathrm{p}$ orbitals and $\mathrm{V}$ $3 \mathrm{~d}$ orbitals in the $\mathrm{CB}$ is expected to greatly limit the migration of electrons to the surface yielding poor electron mobility to the surface, and consequently, poor hydrogen evolution activity as invoked experimentally from the obtained powder bismuth vanadate samples.

\section{Conclusion}

We have examined the effects of intrinsic defects in monoclinic bismuth vanadate $\left(\mathrm{BiVO}_{4}\right)$ on its stability and optoelectronic properties for photochemical water splitting application using DFT and DFPT along with PBE and HSE06 functionals.

Among the various possible explored intrinsic defects, V-enriched structures (containing both $\mathrm{V}$-interstitials and $\mathrm{V}$-antisites on Bi without/with the formation of additional Bi-vacancies) and O-rich structures (containing O-interstitials) were found the most favorable ones. In contrast, Birich structures (containing both Bi-interstitials and Bi-antisites on $\mathrm{V}$ ), Bi-deficient structures (containing Bi-vacancies), and V-deficient structures (containing V-vacancies) were metastable.

We have demonstrated that the energy of $\mathrm{BiVO}_{4}$ band gap can be tuned by the presence of such specific intrinsic defects. This could indeed help find candidate structures that might explain the low hydrogen evolution performance of this material and clarify the discrepancy in the literature. Among the most favorable intrinsic defective structures, only that associated with $\mathrm{V}$-antisites on $\mathrm{Bi}$ with the formation of additional Bi-vacancies (labeled by $\mathrm{Bi}_{(1-5 x)} \mathrm{V}_{(1+3 x)} \mathrm{O}_{4}$ with $x=0.0625)$ revealed narrower band gap energy by about $0.5 \mathrm{eV}$ compared to pristine material 
(calculated value is $2.8 \mathrm{eV}$ ) giving a value of $2.3 \mathrm{eV}$, which is the closest to the experimentally reported ones (in the 2.4-2.6 eV range). This resulted in a red shift of the absorption edge by about $140 \mathrm{~nm}$ as compared to the pristine one. The low electron mobility reported experimentally was confirmed by the relatively large electron effective masses obtained for the intrinsic defective $\mathrm{Bi}_{(1-5 x)} \mathrm{V}_{(1+3 x)} \mathrm{O}_{4}$ (with $x=0.0625$ ) structure along the three principal crystallographic directions. The strongly localized nature of the accommodated electrons on the d-orbitals of the newly substituted $\mathrm{V}$ at $\mathrm{Bi}$ sites was also predicted to be at the origin of the poor hydrogen evolution performance of this material. These findings provide further insights on the interplay between defect modulation and charge carrier transport in metal oxide photoelectrodes, which will help for developing low-cost and efficient solar energy conversion devices.

\section{Associated Content}

\section{Supporting Information}

$\mathrm{S} 1$ : Atomic coordinates for the DFT-optimized structures (based on the $2 \times 1 \times 2$ supercell model) of pristine $\mathrm{BiVO}_{4}$ and the most favorable self-defective $\mathrm{Bi}_{(1-5 x)} \mathrm{V}_{(1+3 x)}(x=0.0625)$ material shown in Figure 2; S2: electronic band structure obtained using the PBE functional for pristine $\mathrm{BiVO}_{4} ; \mathrm{S} 3$ : electronic DOSs obtained using the $\mathrm{PBE}$ functional for pristine $\mathrm{BiVO}_{4}$ without and including the spin-orbit coupling (SOC) effect. This material is available free of charge via the Internet at http://pubs.acs.org. 


\section{Author Information}

\section{Corresponding Author}

*E-mail: moussab.harb@kaust.edu.sa. Phone: +966-012-8080788.

\section{ORCID}

Luigi Cavallo: 0000-0002-1398-338X

Moussab Harb: 0000-0001-5540-9792

\section{Notes}

The authors declare no competing financial interest.

\section{Acknowledgments}

This work was supported by the King Abdullah University of Science and Technology (KAUST). For computer time, this research used the resources of the Supercomputing Laboratory at King Abdullah University of Science and Technology (KAUST) in Thuwal, Saudi Arabia.

\section{References}

(1) Dubal, D. P.; Patil, D. R.; Patil, S. S.; Munirathnam, N. R.; Gomez-Romero, P. BiVO 4 Fern Architectures: A Competitive Anode for Lithium-Ion Batteries. ChemSusChem 2017, 10, 4163-4169.

(2) Han, L.; Abdi, F. F.; van de Krol, R.; Liu, R.; Huang, Z.; Lewerenz, H.-J.; Dam, B.; Zeman, M.; Smets, A. H. M. Efficient Water-Splitting Device Based on a Bismuth Vanadate Photoanode and Thin-Film Silicon Solar Cells. ChemSusChem 2014, 7, 2832-2838.

(3) Pham, T. A.; Ping, Y.; Galli, G. Modelling Heterogeneous Interfaces for Solar Water Splitting. Nat. Mater. 2017, 16, 401-408.

(4) Lin, F.; Wang, D.; Jiang, Z.; Ma, Y.; Li, J.; Li, R.; Li, C. Photocatalytic Oxidation of Thiophene on $\mathrm{BiVO}_{4}$ with Dual co-Catalysts Pt and $\mathrm{RuO}_{2}$ Under Visible Light Irradiation Using Molecular Oxygen as Oxidant. Energy Environ. Sci. 2012, 5, 6400-6406.

(5) Chen, L.; Aarcon-Lado, E.; Hettick, M.; Sharp, I. D.; Lin, Y. J.; Javey, A.; Ager, J. W. Reactive Sputtering of Bismuth Vanadate Photoanodes for Solar Water Splitting. J. Phys. Chem. C 2013, 117, 21635-21642.

(6) Abdi, F. F.; van de Krol, R. Nature and Light Dependence of Bulk Recombination in CoPi-Catalyzed $\mathrm{BiVO}_{4}$ Photoanodes. J. Phys. Chem. C 2012, 116, 9398-9404.

(7) Kudo, A.; Ueda, K.; Kato, H.; Mikami, I. Photocatalytic $\mathrm{O}_{2}$ Evolution Under Visible Light Irradiation on $\mathrm{BiVO}_{4}$ in Aqueous $\mathrm{AgNO}_{3}$ Solution. Catal. Lett. 1998, 53, 229-230. 
(8) Kudo, A.; Omori, K.; Kato, H. A Novel Aqueous Process for Preparation of Crystal Form-Controlled and Highly Crystalline $\mathrm{BiVO}_{4}$ Powder from Layered Vanadates at Room Temperature and its Photocatalytic and Photophysical Properties. J. Am. Chem. Soc. 1999, 121, 11459-11467.

(9) Liu, W.; Cao, L.; Su, G.; Liu, H.; Wang, X.; Zhang, L. Ultrasound Assisted Synthesis of Monoclinic Structured Spindle $\mathrm{BiVO}_{4}$ Particles with Hollow Structure and its Photocatalytic Property. Ultrasonics Sonochem. 2010, 17, 669-674.

(10) Khan, I.; Ali, S.; Mansha, M.; Qurashi, A. Sonochemical Assisted Hydrothermal Synthesis of Pseudo-Flower Shaped Bismuth Vanadate $\left(\mathrm{BiVO}_{4}\right)$ and Their Solar-Driven Water Splitting Application. Ultrasonics Sonochem. 2017, 36, 386-392.

(11) Yu, J. Q.; Zhang, Y.; Kudo, A. Synthesis and Photocatalytic Performances of $\mathrm{BiVO}_{4}$ by Ammonia co-Precipitation Process. J. Solid State Chem. 2009, 182, 223-228.

(12) Xi, G.; Ye, J. Synthesis of Bismuth Vanadate Nanoplates with Exposed $\{001\}$ Facets and Enhanced Visible-Light Photocatalytic Properties. Chem. Commun. 2010, 46, 1893-1895.

(13) A, M.; J, M.; Ashokkumar, M.; Arunachalam, P. A Review on $\mathrm{BiVO}_{4}$ Photocatalyst: Activity Enhancement Methods for Solar Photocatalytic Applications. Appl. Catal. A 2018, 555, 47-74.

(14) Zhou, M.; Bao, J.; Xu, Y.; Zhang, J.; Xie, J.; Guan, M.; Wang, C.; Wen, L.; Lei, Y.; Xie, Y. Photoelectrodes Based Upon $\mathrm{Mo}: \mathrm{BiVO}_{4}$ Inverse Opals for Photoelectrochemical Water Splitting. ACS Nano 2014, 8, 7088-7098.

(15) Yin, W. J.; Wei, S. H.; Al-Jassim, M. M.; Turner, J.; Yan, Y. F., Doping Properties of Monoclinic $\mathrm{BiVO}_{4}$ Studied by First-Principles Density-Functional Theory. Phys. Rev. B 2011, $83,155102 / 1-155102 / 11$.

(16) Jo, W. J.; Jang, J.-W.; Kong, K.-j.; Kang, H. J.; Kim, J. Y.; Jun, H.; Parmar, K. P. S.; Lee, J. S. Phosphate Doping into Monoclinic $\mathrm{BiVO}_{4}$ for Enhanced Photoelectrochemical Water Oxidation Activity. Angew. Chem. Int. Ed. 2012, 51, 3147-3151.

(17) Liang, Y. Q.; Tsubota, T.; Mooij, L. P. A.; van de Krol, R. Highly Improved Quantum Efficiencies for Thin Film BiVO 4 Photoanodes. J. Phys. Chem. C 2011, 115, 17594-17598.

(18) Vinke, I. C.; Diepgrond, J.; Boukamp, B. A.; de Vries, K. J.; Burggraaf, A. J. Bulk and Electrochemical Properties of $\mathrm{BiVO}_{4}$. Solid State Ionics 1992, 57, 83-89.

(19) Rossell, M. D.; Agrawal, P.; Borgschulte, A.; Hébert, C.; Passerone, D.; Erni, R. Direct Evidence of Surface Reduction in Monoclinic BiVO 4 . Chem. Mater. 2015, 27, 3593-3600.

(20) Tayyebi, A.; Soltani, T.; Hong, H.; Lee, B.-K. Improved Photocatalytic and Photoelectrochemical Performance of Monoclinic Bismuth Vanadate by Surface Defect States $\left(\mathrm{Bi}_{1-\mathrm{x}} \mathrm{VO}_{4}\right)$. J. Colloid Interface Sci. 2018, 514, 565-575.

(21) Wang, X.-J.; Zhao, Y.; Li, F.-T.; Dou, L.-J.; Li, Y.-P.; Zhao, J.; Hao, Y.-J. A Chelation Strategy for In-situ Constructing Surface Oxygen Vacancy on $\{001\}$ Facets Exposed BiOBr Nanosheets. Sci. Rep. 2016, 6, 24918/1-24918/11.

(22) Ordon, K.; Kassiba, A.; Makowska-Janusik, M. Electronic, Optical and Vibrational Features of $\mathrm{BiVO}_{4}$ Nanostructures Investigated by First-Principles Calculations. RSC Adv. 2016, 6, 110695-110705.

(23) Wadnerkar, N.; English, N. J. Density Functional Theory Investigations of Bismuth Vanadate: Effect of Hybrid Functionals. Comput. Mater. Sci. 2013, 74, 33-39.

(24) Harb, M. Predicting Suitable Optoelectronic Properties of Monoclinic VON Semiconductor Crystals for Photovoltaics Using Accurate First-Principles Computations. Phys. Chem. Chem. Phys. 2015, 17, 25244-25249. 
(25) Harb, M.; Cavallo, L.; Basset, J.-M. Major Difference in Visible-Light Photocatalytic Features Between Perfect and Self-Defective $\mathrm{Ta}_{3} \mathrm{~N}_{5}$ Materials: A Screened Coulomb Hybrid DFT Investigation. J. Phys. Chem. C 2014, 118, 20784-20790.

(26) Kresse, G.; Furthmüller, J. Efficiency of Ab-Initio Total Energy Calculations for Metals and Semiconductors Using a Plane-Wave Basis Set. Comput. Mater. Sci. 1996, 6, 15-50.

(27) Kresse, G.; Furthmüller, J. Efficient Iterative Schemes for Ab Initio Total-Energy Calculations Using a Plane-Wave Basis Set. Phys. Rev. B 1996, 54, 11169-11186.

(28) Kresse, G.; Hafner, J. Ab Initio Molecular-Dynamics Simulation of the LiquidMetalamorphous-Semiconductor Transition in Germanium. Phys. Rev. B 1994, 49, 14251-14269. (29) Perdew, J. P.; Burke, K.; Ernzerhof, M. Generalized Gradient Approximation Made Simple. Phys. Rev. Lett. 1996, 77, 3865-3868.

(30) Blöchl, P. E., Projector Augmented-Wave Method. Phys. Rev. B 1994, 50, 17953-17979.

(31) Monkhorst, H. J.; Pack, J. D., Special Points for Brillouin-Zone Integrations. Phys. Rev. $B$ 1976, 13, 5188-5192.

(32) Jang, J.-W.; Friedrich, D.; Müller, S.; Lamers, M.; Hempel, H.; Lardhi, S.; Cao, Z.; Harb, M.; Cavallo, L.; Heller, R., et al. Enhancing Charge Carrier Lifetime in Metal Oxide Photoelectrodes through Mild Hydrogen Treatment. Adv. Energy Mater. 2017, 7, 1701536/1$1701536 / 11$.

(33) Harb, M.; Cavallo, L. Suitable Fundamental Properties of $\mathrm{Ta}_{0.75} \mathrm{~V}_{0.25} \mathrm{ON}$ Material for Visible-Light-Driven Photocatalysis: A DFT Study. ACS Omega 2016, 1, 1041-1048.

(34) Harb, M. First-Principles Investigation of Optoelectronic and Redox Properties of $\left(\mathrm{Ta}_{1-}\right.$ $\left.{ }_{x} \mathrm{Nb}_{\mathrm{x}}\right)$ ON Compounds for Photocatalysis. J. Phys. Chem. C 2015, 119, 4565-4572.

(35) Hafner, J. Ab-initio Simulations of Materials using VASP: Density-Functional Theory and Beyond. J. Comput. Chem. 2008, 29, 2044-2078.

(36) Heyd, J.; Scuseria, G. E.; Ernzerhof, M. Hybrid Functionals Based on a Screened Coulomb potential. J. Chem. Phys. 2003, 118, 8207-8215.

(37) Lardhi, S.; Noureldine, D.; Harb, M.; Ziani, A.; Cavallo, L.; Takanabe, K. Determination of the Electronic, Dielectric, and Optical properties of Sillenite $\mathrm{Bi}_{12} \mathrm{TiO}_{20}$ and Perovskite-Like $\mathrm{Bi}_{4} \mathrm{Ti}_{3} \mathrm{O}_{12}$ Materials from Hybrid First-Principle Calculations. J. Chem. Phys. 2016, 144, 134702/1-134702/8.

(38) Noureldine, D.; Lardhi, S.; Ziani, A.; Harb, M.; Cavallo, L.; Takanabe, K. Combined Experimental-Theoretical Study of the Optoelectronic Properties of Non-Stoichiometric Pyrochlore Bismuth Titanate. J. Mater. Chem. C 2015, 3, 12032-12039.

(39) Lardhi, S.; Curutchet, A.; Cavallo, L.; Harb, M.; Le Bahers, T. Ab Initio Assessment of $\mathrm{Bi}_{1-\mathrm{x}} \mathrm{RE}_{\mathrm{x}} \mathrm{CuOS}(\mathrm{RE}=\mathrm{La}, \mathrm{Gd}, \mathrm{Y}, \mathrm{Lu})$ Solid Solutions as a Semiconductor for Photochemical Water Splitting. Phys. Chem. Chem. Phys. 2017, 19, 12321-12330.

(40) Ullah, H.; Tahir, A. A.; Mallick, T. K. Structural and Electronic Properties of Oxygen Defective and Se-Doped p-Type $\mathrm{BiVO}_{4}(001)$ Thin Film for the Applications of Photocatalysis. Appl. Catal. B: Environ. 2018, 224, 895-903.

(41) Sleight, A. W.; Chen, H. Y.; Ferretti, A.; Cox, D. E. Crystal-Growth and Structure of BiVO $_{4}$. Mater. Res. Bull. 1979, 14, 1571-1581.

(42) Zhao, Z.; Li, Z.; Zou, Z. Electronic Structure and Optical Properties of Monoclinic Clinobisvanite $\mathrm{BiVO}_{4}$. Phys. Chem. Chem. Phys. 2011, 13, 4746-4753.

(43) Zhao, Z.; Luo, W.; Li, Z.; Zou, Z. Density Functional Theory Study of Doping Effects in Monoclinic Clinobisvanite BiVO 4 . Phys. Lett. A 2010, 374, 4919-4927. 
(44) Cooper, J. K.; Gul, S.; Toma, F. M.; Chen, L.; Liu, Y.-S.; Guo, J.; Ager, J. W.; Yano, J.; Sharp, I. D. Indirect Bandgap and Optical Properties of Monoclinic Bismuth Vanadate. J. Phys. Chem. C 2015, 119, 2969-2974.

(45) Ghamri, J.; Baussart, H.; Bras, M. 1.; Leroy, J.-M. Spectroscopic Study of $\mathrm{Bi}_{\mathrm{x}} \mathrm{Eu}_{1-\mathrm{x}} \mathrm{VO}_{4}$ and $\mathrm{Bi}_{\mathrm{y}} \mathrm{Gd}_{1-\mathrm{y}} \mathrm{VO}_{4}$ Mixed Oxides. J. Phys. Chem. Solid 1989, 50, 1237-1244.

(46) Park, Y.; McDonald, K. J.; Choi, K.-S. Progress in Bismuth Vanadate Photoanodes for use in Solar Water Oxidation. Chem. Soc. Rev. 2013, 42, 2321-2337.

(47) Tokunaga, S.; Kato, H.; Kudo, A. Selective Preparation of Monoclinic and Tetragonal $\mathrm{BiVO}_{4}$ with Scheelite Structure and Their Photocatalytic Properties. Chem. Mater. 2001, 13, 4624-4628.

(48) Kim, T. W.; Ping, Y.; Galli, G. A.; Choi, K.-S., Simultaneous Enhancements in Photon Absorption and Charge Transport of Bismuth Vanadate Photoanodes for Solar Water Splitting. Nat. Commun. 2015, 6, 8769/1-8769/10.

(49) Barad, Y.; Gopalan, V. In-Situ Probing of Domain Poling in $\mathrm{Bi}_{4} \mathrm{Ti}_{3} \mathrm{O}_{12}$ Thin Films by Optical Second Harmonic Generation. Integr. Ferroelectrics 2002, 44, 19-24.

(50) Bahers, T. L.; Rérat, M.; Sautet, P. Semiconductors Used in Photovoltaic and Photocatalytic Devices: Assessing Fundamental Properties from DFT. J. Phys. Chem. C 2014, 118, 5997-6008.

(51) Kweon, K. E.; Hwang, G. S.; Kim, J.; Kim, S.; Kim, S. Electron Small Polarons and their Transport in Bismuth Vanadate: a First Principles Study. Phys. Chem. Chem. Phys. 2015, 17, 256-260.

(52) Tang, H.; Prasad, K.; Sanjines, R.; Schmid, P. E.; Levy, F. Electrical and OpticalProperties of $\mathrm{TiO}_{2}$ Anatase Thin-Films. J. Appl. Phys. 1994, 75, 2042-2047.

(53) Pascual, J.; Camassel, J.; Mathieu, H. Resolved Quadrupolar Transition in $\mathrm{TiO}_{2}$. Phys. Rev. Lett. 1977, 39, 1490-1493.

(54) Enright, B.; Fitzmaurice, D. Spectroscopic Determination of Electron and Hole Effective Masses in a Nanocrystalline Semiconductor Film. J. Phys. Chem. 1996, 100, 1027-1035.

(55) Singh, A. P.; Kodan, N.; Mehta, B. R.; Held, A.; Mayrhofer, L.; Moseler, M. Band Edge Engineering in $\mathrm{BiVO}_{4} / \mathrm{TiO}_{2}$ Heterostructure: Enhanced Photoelectrochemical Performance through Improved Charge Transfer. ACS Catal. 2016, 6, 5311-5318.

(56) Yan, L.; Zhao, W.; Liu, Z. F. 1D ZnO/BiVO 4 Heterojunction Photoanodes for Efficient Photoelectrochemical Water Splitting. Dalton Trans. 2016, 45, 11346-11352. 


\section{TOC Graphic}

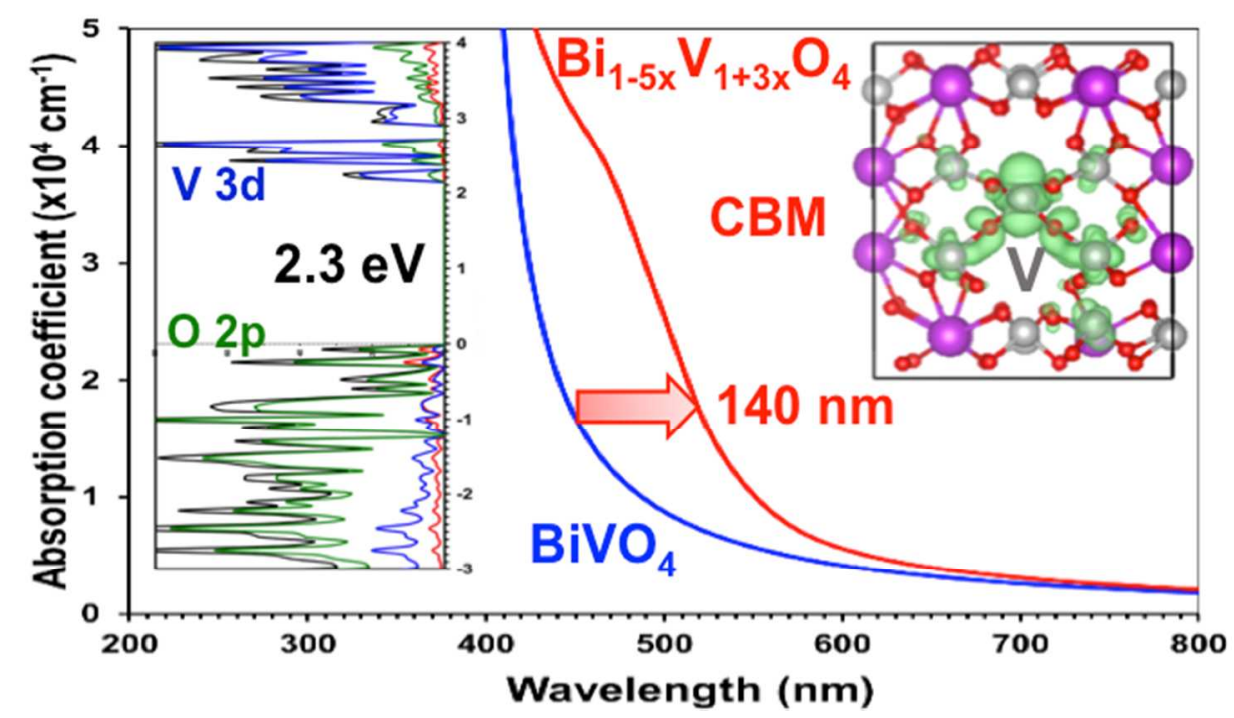

